\title{
Research on Safety Monitoring Behavior Recognition System Based on Video
}

\author{
Ming Li \\ Lanzhou Resource and Environment Voc-Tech College, Electrical and Mechanical Department, Lanzhou \\ 730021, China.
}

Keywords: Video, Coal Mine, Safety Monitoring, Identification System.

\begin{abstract}
Coal mine is an important way of energy mining in China. In the process of coal mining, safety management is an important requirement for coal mining work to be carried out in an orderly manner. With the rapid development of science and technology, the video security monitoring system based on information technology provides a powerful help for coal mine safety. The video security monitoring system can collect, analyze and process the monitoring target according to the coal mine safety standard, thus forming a comprehensive coverage safety monitoring system. Based on the video, this paper aims to improve the overall safety of coal mine operation and reduce the safety accidents in coal mine production by means of information technology.
\end{abstract}

\section{Introduction}

With the popularization of information technology in all fields of society, it provides a powerful support for coal mine safety monitoring and management. The video technology mode in information technology can collect and process the video data for the whole process of coal mine production, and rely on the computer system to transform the information data into visual image, so that the coal mine safety management can form an intelligent, high-efficiency and visual integrated control system. The safety monitoring management based on video is mainly based on the advantage of video image acquisition to monitor the mine operation in an all-round way. The video, like the eyes of the computer, converts and compresses the monitored images, forms a program language that the computer can read, transmits it to the central system of coal mine safety management through the network transmission environment, and uses the analysis and judgment ability of the intelligent technology to identify, so as to achieve the overall supervision goal of coal mine operation safety.

\section{The Function of Coal Mine Safety Monitoring Behavior Identification System}

\subsection{Intelligent Behavior Recognition Function}

When the safety monitoring behavior of coal mine is identified, the system function needs to have the identification map database of the behavior characteristics, to ensure that the data in the video can be compared with the established behavior, and to realize the early warning of the violation behavior. Under the mode of behavior recognition and early warning, the mine environment is monitored in real time by the recognition device pre-located in the mine, and the data content is processed and analyzed, so that the information data can be formed by video technology as the monitoring medium. For the database information of behavior identification, it is necessary to update it in time, and store the behavior data through the implementation process of coal mine operation[1]. Coal production safety management personnel should communicate closely with information technology personnel, refine and express the behavior of coal mine safety monitoring through computer language, and store it in the identification system continuously. When the video device collects the behavior which does not conform to the security standard, the behavior identification basis of the security management is compared and judged by the comparison information obtained by the database. 


\subsection{Comprehensive Information Management Function}

Video system can play a comprehensive and effective monitoring and management role in the process of coal mine operation. Video surveillance information is the data collection of all behavior and environment in the production process. The comprehensive information management includes multi-level and multi-angle information system such as historical data, real-time data, monitoring parameters and early warning setting. During the period of coal mine information existence and the whole production, through the comprehensive management of information, it can rely on the information collected by monitoring equipment to help the coal mine safety management personnel to check repeatedly, and can also use the information data content to restore the coal mine production link, so as to realize the purpose of remote control of coal mine safety. Coal mine information monitoring parameters is a basic monitoring standard design, which can ensure the accuracy of information system in behavior identification and improve the level of coal mine safety management.

\subsection{Systematic Information Statistics Function}

In the coal mine information management system, the safety management basis of the system is established through the identification system, and the behavior identification should be fed back to the department where the perpetrator is located. Therefore, the information of the coal mine workers and departments should be systematically managed in the identification system, and the work role, working hours and working position of the relevant personnel should be collected in the behavior identification system.

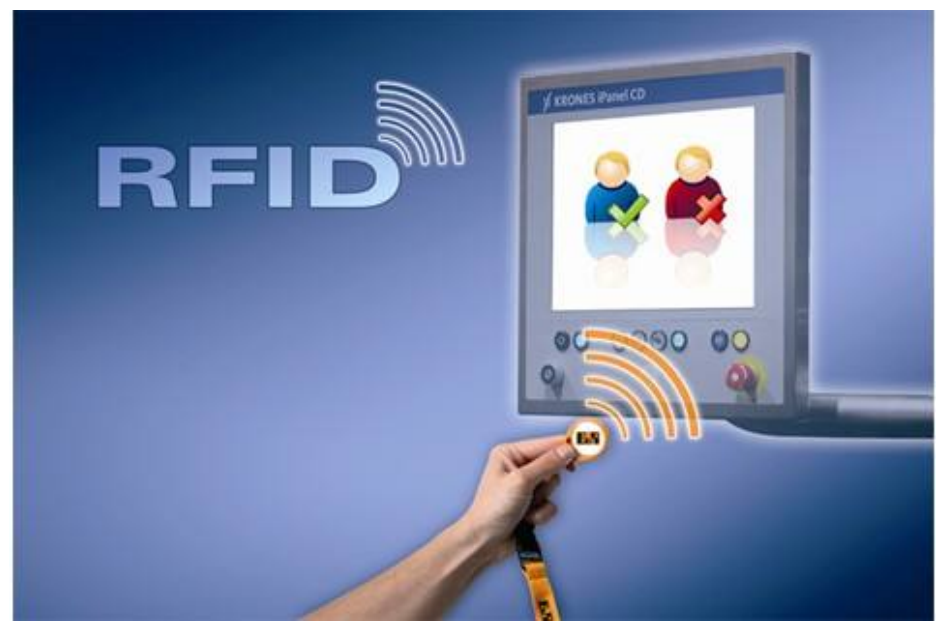

Figure 1 Identification system function chart

\section{Design of Coal Mine Safety Monitoring Behavior Identification System}

\subsection{Design of Database Architecture for Behavior Recognition System}

In the process of coal mine safety monitoring, it is necessary to design the database architecture of the behavior identification system. Database architecture is the basic database based on computer software development, the database needs to establish a framework system to store all the information in coal mine operations. The process of coal mine operation is more complex, involving a large number of personnel and machinery, when storing relevant information content, it should be classified according to different behavior identification needs[2]. The database has the powerful function of data relation management, in the process of coal mine production, the information collected based on video is relatively large, in order to improve the efficiency of database access, we can set up a special database server, which is dedicated to serving the database of the behavior recognition system, so that the information capacity and the speed of information processing can be guaranteed to the maximum extent. In the database architecture design, the data content collected by the video should be standardized and named, and the naming principle can adopt the comprehensive naming methods such as time, path and behavior characteristics, so that the behavior recognition 
system can get the data content quickly and accurately when calling these data.

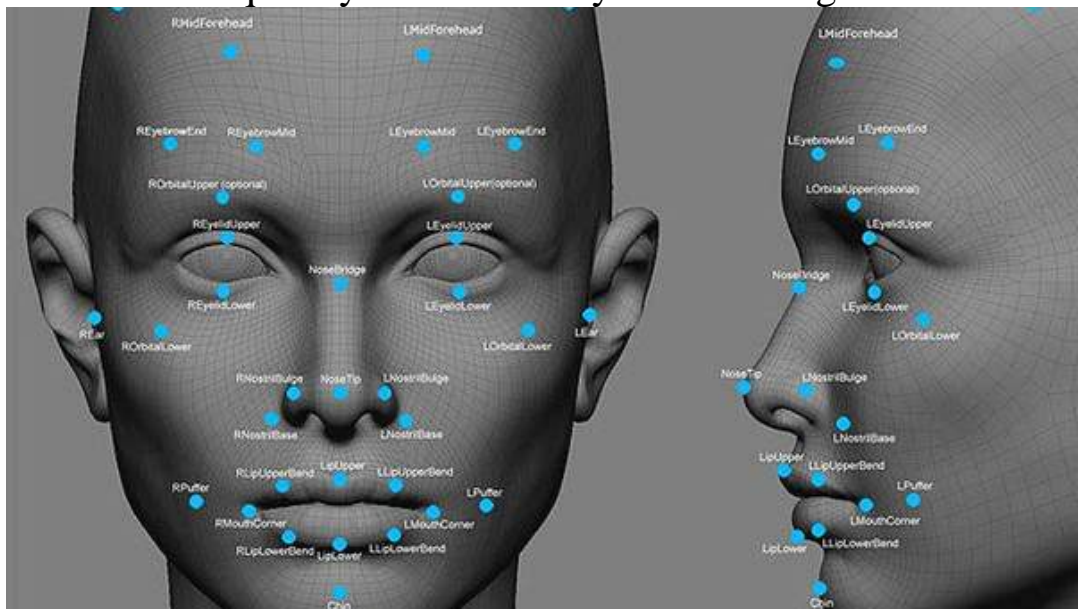

Figure 2 Identification system architecture

\subsection{Program Server Design for Behavior Recognition System}

The coal mine safety management behavior identification system is based on the video data information processing and analysis, in the information technology as the medium monitoring process, needs to use the program to establish the computer language system, realizes the safety management instruction identification and the execution. The computer system program relies on the running of the program server to separate the graphics module from the control layer module in the program design, so that the view type data collected by the video device can be logically processed in the computer program, and the processed information can be passed to the control layer[3]. The control layer opens the operation right on the basis of data exchange, and the computer system automatically executes the control instruction, so that the coal mine safety management behavior identification system can respond to the safety instruction accordingly, and realize the safety service goal of the system program.

\subsection{Operation Page Architecture Design of Behavior Recognition System}

The operation page of coal mine safety management behavior identification system is the basis of human-computer interaction. In coal mine management, managers are required to monitor the production process, but managers cannot issue work instructions through abstract computer language, which requires a bridge between computer system and personnel, and the operable page is the technical means to achieve this goal. The design of the operation page architecture in the behavior recognition system is to express the abstract system function through the visual and visual page function, so that the coal mine safety management personnel can operate the system directly according to the page text and graphics in the use behavior recognition system[4]. In today's mature information technology, the computer front-end interface can realize the operation requirements of coal mine safety management through a large number of functional components, and bring good operation experience to managers with its strong visibility and maneuverability. Even if the safety managers do not have professional information system design skills, they can effectively implement the operation flow of the identification system and enhance the applicability of the behavior identification system.

\subsection{Design of Behavior Recognition System Analysis Engine}

The purpose of the coal mine safety management system is to analyze the safety of every link of coal mine production, which requires the system to have good data processing and analysis performance. The behavior recognition system can be divided into the detection and identification of the coal mine working environment according to the type of identification, the identification of the coal mine working process, and the identification of the coal mine operators. The identification target detection in coal mine identification is the basis of all behavior recognition process. The data analysis engine is established through the behavior recognition system, which can extract the coal 
mine operation environment information collected by the video equipment under the model condition, so as to judge whether the personnel in the video screen are the coal mine workers, whether the operation behavior of the staff is in accordance with the regulations, and whether the coal mine operation environment meets the safety production conditions, etc. The behavior recognition model based on the analysis engine is mainly to extract the feature of the video picture, and analyze whether the travel is the safety specification of the identification target from the point of view of the system definition through the standardized comparison of the extracted target features.

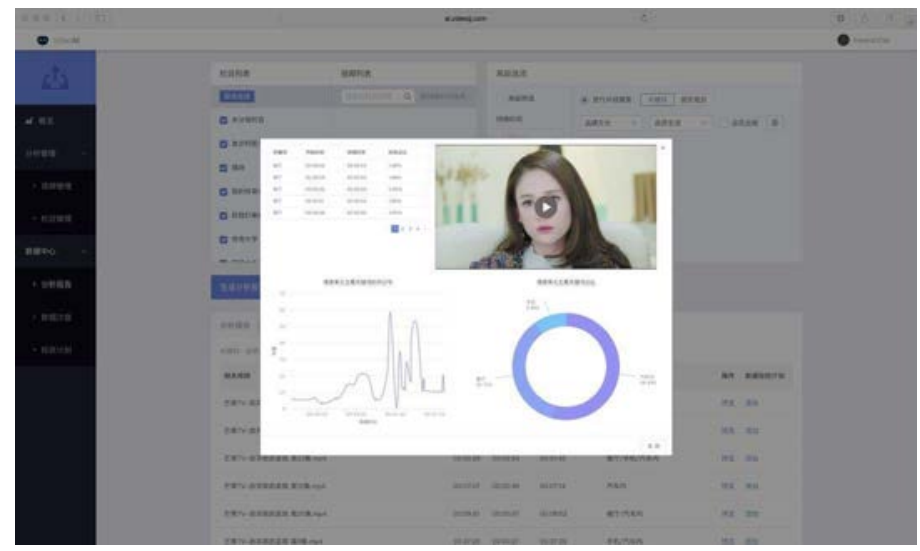

Figure 3 Identification analysis chart

\subsection{Early Warning Design of Behavior Identification System}

The early warning design of the behavior recognition system is based on the split frame analysis of the video picture information. The minimum unit in the computer picture is one frame, and the coal mine video picture is divided into continuous frames in the process of data processing. Through the early warning design of the behavior recognition system, each frame is modeled and analyzed, and the behavior target of each frame is calculated by computer algorithm. Early warning design can help managers focus on dealing with important differential behavior, and focus on checking the safety risk of these behaviors, and improve the safety monitoring ability of coal mine operation.

\section{Conclusion}

Coal mine operation is a high safety risk factor work content, in the information technology based video surveillance environment, through the information technology means to establish a comprehensive safety management system, the whole process of coal mine operation and all personnel into the unified management system. The behavior recognition system is based on video, through the video information acquisition device set up in the mine to obtain all the information in the mine, and through the behavior recognition system to process and analyze the information, to provide a strong support for the safety management of the mine.

\section{Acknowledgements}

Research project of Gansu Higher Education Institute, project number: 2018C-25.

\section{References}

[1] Big, hair. Research on data anomaly identification technology of monitoring points in coal mine safety monitoring system. Mining Machinery, vol. 41, no. 4, pp. 120-123, 2013.

[2] Tiandi (Changzhou) Automation Co., Ltd., China Coal Science and Technology Group Changzhou Research Institute Co., Ltd. A method for automatic identification and plug and play of coal mine safety monitoring system, 2018 
[3] Tiandi (Changzhou) Automation Co., Ltd., China Coal Science and Technology Group Changzhou Research Institute Co., Ltd., Yanzhou Coal Industry Co., Ltd. A method of repeated identification and alarm of sensor address in coal mine safety monitoring system, 2017

[4] Shanxi Elite Technology Co., Ltd. A gas false alarm identification method based on coal mine safety monitoring network system, 2018. 